PROCEEDINGS OF THE

AMERICAN MATHEMATICAL SOCIETY

Volume 140, Number 4, April 2012, Pages 1281-1295

S 0002-9939(2011)11060-8

Article electronically published on July 22, 2011

\title{
UNITARY EQUIVALENCE TO A TRUNCATED TOEPLITZ OPERATOR: ANALYTIC SYMBOLS
}

\author{
STEPHAN RAMON GARCIA, DANIEL E. POORE, AND WILLIAM T. ROSS
}

(Communicated by Richard Rochberg)

\begin{abstract}
Unlike Toeplitz operators on $H^{2}$, truncated Toeplitz operators do not have a natural matricial characterization. Consequently, these operators are difficult to study numerically. In this paper we provide criteria for a matrix with distinct eigenvalues to be unitarily equivalent to a truncated Toeplitz operator having an analytic symbol. This test is constructive, and we illustrate it with several examples. As a byproduct, we also prove that every complex symmetric operator on a Hilbert space of dimension $\leq 3$ is unitarily equivalent to a direct sum of truncated Toeplitz operators.
\end{abstract}

\section{INTRODUCTION}

Interest in truncated Toeplitz operators has blossomed over the last several years [4, 5, 8, 7, 15, 26, 27, 28, 29, 31, sparked by a seminal paper of D. Sarason [26. Unfortunately, only the very simplest truncated Toeplitz operators, the finite Toeplitz matrices, have any sort of practical matricial description. Thus the numerical study of truncated Toeplitz operators is difficult, even in low dimensions. For instance, while the theory of pseudospectra for Toeplitz matrices has recently undergone spirited development [33, Ch. II], many basic questions about truncated Toeplitz operators remain unanswered. For instance, finding a characterization of rank-two self-adjoint truncated Toeplitz operators is still an open problem [26, p. 508] despite the fact that all rank-one truncated Toeplitz operators have already been identified [26, Thm. 5.1].

Although a few results concerning matrix representations of truncated Toeplitz operators have been obtained [7, 8, 31, the general question of determining whether a given matrix represents a truncated Toeplitz operator, with respect to some orthonormal basis, appears difficult. On the other hand, it is known that every truncated Toeplitz operator is unitarily equivalent to a complex symmetric matrix 11, 13, a somewhat more general issue which has been studied by several authors [3, 12, 32, 34].

Our work is partly motivated by the question of whether truncated Toeplitz operators serve as some sort of model for complex symmetric operators. A significant

Received by the editors December 21, 2010.

2010 Mathematics Subject Classification. Primary 47A05, 47B35, 47 B99.

Key words and phrases. Toeplitz operator, model space, truncated Toeplitz operator, reproducing kernel, complex symmetric operator, conjugation, hyperbolic geometry, Euclid, Hilbert's axioms, pseudo-hyperbolic metric, hyperbolic metric, Poincaré model, trace.

The first author was partially supported by National Science Foundation Grant DMS-1001614.

(C)2011 American Mathematical Society Reverts to public domain 28 years from publication 
amount of evidence has been produced in this direction, starting with D. Sarason's early observation that the Volterra integration operator is unitarily equivalent to a truncated Toeplitz operator [25] (see also [23, p. 41]). Since then, many other examples of complex symmetric operators which are representable in terms of truncated Toeplitz operators have emerged. For instance, normal operators [8, Thm. 5.6], rank-one operators, [8, Thm. 5.1], $2 \times 2$ matrices [8, Thm. 5.2], and inflations of finite Toeplitz matrices [8, Thm. 5.7] are unitarily equivalent to truncated Toeplitz operators. The recent preprint 31 contains a host of other examples.

Before stating our main results, we briefly review some of the necessary preliminaries. Let $H^{2}$ denote the Hardy space on the open unit disk $\mathbb{D}$, let $H^{\infty}$ denote the Banach algebra of all bounded analytic functions on $\mathbb{D}$, and let $L^{\infty}:=L^{\infty}(\partial \mathbb{D})$ and $L^{2}:=L^{2}(\partial \mathbb{D})$ denote the usual Lebesgue spaces on the unit circle $\partial \mathbb{D}[9,19$. To each nonconstant inner function $\Theta$ we associate the model space $\mathcal{K}_{\Theta}:=H^{2} \ominus \Theta H^{2}$, which is the reproducing kernel Hilbert space corresponding to the kernel

$$
K_{\lambda}(z):=\frac{1-\overline{\Theta(\lambda)} \Theta(z)}{1-\bar{\lambda} z}, \quad z, \lambda \in \mathbb{D}
$$

For our purposes, we find it more convenient to work with the normalized kernels $k_{\lambda}:=\sqrt{\frac{1-|\lambda|^{2}}{1-|\Theta(\lambda)|^{2}}} K_{\lambda}$. The space $\mathcal{K}_{\Theta}$ carries a natural conjugation (an isometric, conjugate-linear, involution)

$$
C f:=\overline{f z} \Theta,
$$

defined in terms of boundary functions [11, 13, 15. The normalized conjugate kernels $\tilde{k}_{\lambda}:=C k_{\lambda}$ are of particular interest to us.

For each symbol $\varphi$ in $L^{2}$ the corresponding truncated Toeplitz operator $A_{\varphi}$ is the densely defined operator on $\mathcal{K}_{\Theta}$ given by

$$
A_{\varphi} f:=P_{\Theta}(\varphi f) .
$$

When we wish to be specific about the inner function $\Theta$, we often write $A_{\varphi}^{\Theta}$ in place of $A_{\varphi}$. The adjoint of $A_{\varphi}$ is the operator $A_{\bar{\varphi}}$, and it is easy to see that $A_{\varphi}=C A_{\varphi}^{*} C$, where $C$ denotes the conjugation (11). In other words, $A_{\varphi}$ is a complex symmetric operator [11, 13, 14, and hence it can be represented as a complex symmetric (i.e., self-transpose) matrix with respect to some orthonormal basis of $\mathcal{K}_{\Theta}$ [11] (see also [15, Sect. 5.2]).

We are most interested in the case where $\varphi \in H^{\infty}$ and $\Theta$ is a finite Blaschke product having distinct zeros $z_{1}, z_{2}, \ldots, z_{n}$. In this case we have

$$
k_{z_{i}}=\frac{\sqrt{1-\left|z_{i}\right|^{2}}}{1-\overline{z_{i}} z}, \quad \tilde{k}_{z_{i}}=\frac{\sqrt{1-\left|z_{i}\right|^{2}} \Theta(z)}{z-z_{i}} .
$$

For each $\varphi$ in $H^{\infty}$ the eigenvalues of the analytic truncated Toeplitz operator $A_{\varphi}^{\Theta}$ are given by $\varphi\left(z_{1}\right), \varphi\left(z_{2}\right), \ldots, \varphi\left(z_{n}\right)$, with corresponding normalized eigenvectors $\tilde{k}_{z_{1}}, \tilde{k}_{z_{2}}, \ldots, \tilde{k}_{z_{n}}$ [1, p. 10]. In particular, nonzero eigenvectors of an analytic truncated Toeplitz operator are never orthogonal to each other.

One of the main results of this paper is the following simple criterion for determining whether or not a given matrix is unitarily equivalent to a trunctated Toeplitz operator having an analytic symbol. 
Theorem 1.1. If $M \in \mathbf{M}_{n}(\mathbb{C})$ has distinct eigenvalues $\lambda_{1}, \lambda_{2}, \ldots, \lambda_{n}$ with corresponding unit eigenvectors $\mathbf{x}_{1}, \mathbf{x}_{2}, \ldots, \mathbf{x}_{n}$, then $M$ is unitarily equivalent to an analytic truncated Toeplitz operator if and only if there exist distinct points $z_{1}, z_{2}, \ldots$, $z_{n-1}$ in $\mathbb{D}$ such that

$$
\left\langle\mathbf{x}_{n}, \mathbf{x}_{i}\right\rangle\left\langle\mathbf{x}_{i}, \mathbf{x}_{j}\right\rangle\left\langle\mathbf{x}_{j}, \mathbf{x}_{n}\right\rangle=\frac{\left(1-\left|z_{i}\right|^{2}\right)\left(1-\left|z_{j}\right|^{2}\right)}{1-\overline{z_{j}} z_{i}}
$$

holds for $1 \leq i \leq j<n$.

The method of Theorem 1.1 is constructive in the sense that if (3) is satisfied, then we can construct an inner function $\Theta$ and a polynomial $\varphi$ such that $M$ is unitarily equivalent to $A_{\varphi}^{\Theta}$ (denoted $M \cong A_{\varphi}^{\Theta}$ ). In fact, $\Theta$ is the Blaschke product having zeros at $z_{1}, z_{2}, \ldots, z_{n-1}$ and $z_{n}=0$.

Although condition (3) appears somewhat complicated, it encodes a wealth of geometric data. For instance, setting $j=i$ in (3) yields $\left|\left\langle\mathbf{x}_{i}, \mathbf{x}_{n}\right\rangle\right|^{2}=1-\left|z_{i}\right|^{2}$. This in turn provides us with the formula

$$
\left|z_{i}\right|=\sqrt{1-\left|\left\langle\mathbf{x}_{i}, \mathbf{x}_{n}\right\rangle\right|^{2}}
$$

for the moduli of the unknown points $z_{1}, z_{2}, \ldots, z_{n-1}$. Furthermore, the proof of Theorem 1.1 actually implies that

$$
\left|\left\langle\mathbf{x}_{i}, \mathbf{x}_{j}\right\rangle\right|^{2}=\left|\left\langle k_{z_{i}}, k_{z_{j}}\right\rangle\right|^{2}=1-\rho^{2}\left(z_{i}, z_{j}\right),
$$

where

$$
\rho(z, w):=\left|\frac{z-w}{1-\bar{w} z}\right|
$$

denotes the pseudohyperbolic metric on $\mathbb{D}$. In other words, we can obtain the pseudohyperbolic distances $\rho\left(z_{i}, z_{j}\right)$ directly from the data $\mathbf{x}_{1}, \mathbf{x}_{2}, \ldots, \mathbf{x}_{n}$ :

$$
\rho\left(z_{i}, z_{j}\right)=\sqrt{1-\left|\left\langle\mathbf{x}_{i}, \mathbf{x}_{j}\right\rangle\right|^{2}} .
$$

Let us briefly summarize the contents of this paper. The proof of Theorem 1.1 is contained in Section 22 In Section 3, we use elementary hyperbolic geometry and some of the preceding geometric observations to construct an illuminating example. Sections 4 and 5 provide complete analyses of the $2 \times 2$ and $3 \times 3$ cases, respectively. Among other things, we prove that every complex symmetric operator on a three-dimensional Hilbert space is unitarily equivalent to a direct sum of truncated Toeplitz operators (Theorem [5.2). Section 6 concerns a simple necessary condition for a matrix to be unitarily equivalent to an analytic truncated Toeplitz operator. We conclude this paper in Section 7 with a number of open problems that we hope will spur further research into this topic.

\section{Proof of Theorem 1.1}

To prove Theorem 1.1, we require a few preliminaries. We first note that for each disk automorphism $\psi$, the weighted composition operator $U_{\psi}: \mathcal{K}_{\Theta} \rightarrow \mathcal{K}_{\Theta \circ \psi}$ defined by $U_{\psi} f:=\sqrt{\psi^{\prime}}(f \circ \psi)$ is unitary and furnishes a bijection between the set of analytic truncated Toeplitz operators on $\mathcal{K}_{\Theta}$ and those on $\mathcal{K}_{\Theta \circ \psi}$. In particular,

$$
A_{\varphi}^{\Theta} \cong A_{\varphi \circ \psi}^{\Theta \circ \psi}
$$

holds for all $\varphi$ in $H^{\infty}$ [8, Prop. 4.1]. Our next ingredient is the following simple lemma, which is inspired by the proof of [3, Thm. 2]. 
Lemma 2.1. Let $\mathcal{X}$ and $\mathcal{Y}$ be $n$-dimensional Hilbert spaces. If $x_{1}, x_{2}, \ldots, x_{n} \in$ $\mathcal{X}$ and $y_{1}, y_{2}, \ldots, y_{n} \in \mathcal{Y}$ are linearly independent sets of unit vectors such that $\left\langle y_{i}, y_{j}\right\rangle \neq 0$ for $1 \leq i, j \leq n$, then the following are equivalent:

(i) There exist unimodular constants $\alpha_{1}, \alpha_{2}, \ldots, \alpha_{n}$ and a unitary operator $U: \mathcal{X} \rightarrow \mathcal{Y}$ such that $U x_{i}=\alpha_{i} y_{i}$ for $1 \leq i \leq n$.

(ii) There exist unimodular constants $\alpha_{1}, \alpha_{2}, \ldots, \alpha_{n}$ such that

$$
\left\langle x_{i}, x_{j}\right\rangle=\alpha_{i} \overline{\alpha_{j}}\left\langle y_{i}, y_{j}\right\rangle
$$

for $1 \leq i \leq j \leq n$.

(iii) For some fixed $k$,

$$
\left\langle x_{k}, x_{i}\right\rangle\left\langle x_{i}, x_{j}\right\rangle\left\langle x_{j}, x_{k}\right\rangle=\left\langle y_{k}, y_{i}\right\rangle\left\langle y_{i}, y_{j}\right\rangle\left\langle y_{j}, y_{k}\right\rangle
$$

holds for $1 \leq i \leq j \leq n$.

(iv) Condition (17) holds for $1 \leq i \leq j \leq k \leq n$.

Proof. The implications (i) $\Leftrightarrow$ (ii) $\Rightarrow$ (iv) $\Rightarrow$ (iii) are obvious. We therefore prove only (iii) $\Rightarrow$ (ii). Setting $j=k$ in (7) reveals that $\left|\left\langle x_{i}, x_{k}\right\rangle\right|=\left|\left\langle y_{i}, y_{k}\right\rangle\right| \neq 0$ for $1 \leq i \leq n$. In light of this, we conclude from (77) that $\left|\left\langle x_{i}, x_{j}\right\rangle\right|=\left|\left\langle y_{i}, y_{j}\right\rangle\right| \neq 0$ for $1 \leq i, j \leq n$, whence the constants

$$
\beta_{i j}=\frac{\left\langle x_{i}, x_{j}\right\rangle}{\left\langle y_{i}, y_{j}\right\rangle}
$$

are unimodular and satisfy $\beta_{i j}=\beta_{i k} \beta_{k j}$ for $1 \leq i, j \leq n$. Now define $\alpha_{i}=\beta_{i k}$ for $1 \leq i \leq n$ and observe that

$$
\left\langle x_{i}, x_{j}\right\rangle=\beta_{i j}\left\langle y_{i}, y_{j}\right\rangle=\beta_{i k} \beta_{k j}\left\langle y_{i}, y_{j}\right\rangle=\alpha_{i} \overline{\alpha_{j}}\left\langle y_{i}, y_{j}\right\rangle .
$$

With additional effort, one can remove the hypothesis that $\left\langle y_{i}, y_{j}\right\rangle \neq 0$ for $1 \leq$ $i, j \leq n$. However, this is unnecessary in our case since eigenvectors of an analytic truncated Toeplitz operator cannot be orthogonal to each other.

Proof of Theorem 1.1. $(\Rightarrow)$ Suppose that $U M=A_{\varphi}^{\Theta} U$, where $\varphi \in H^{\infty}$ and $U$ : $\mathbb{C}^{n} \rightarrow \mathcal{K}_{\Theta}$ is unitary. Next observe that if $M \mathbf{x}_{i}=\lambda_{i} \mathbf{x}_{i}$, then $A_{\varphi}^{\Theta}\left(U \mathbf{x}_{i}\right)=\lambda_{i}\left(U \mathbf{x}_{i}\right)$ for $1 \leq i \leq n$. Thus there exists an enumeration $z_{1}, z_{2}, \ldots, z_{n}$ of the zeros of $\Theta$ and unimodular constants $\alpha_{1}, \alpha_{2}, \ldots, \alpha_{n}$ so that $U \mathbf{x}_{i}=\alpha_{i} \tilde{k}_{z_{i}}$ for $1 \leq i \leq n$. Without loss of generality we may assume that $z_{n}=0$. Indeed, otherwise let $\psi$ be an automorphism of $\mathbb{D}$ satisfying $\psi(0)=z_{n}$ and note that $M \cong A_{\varphi}^{\Theta} \cong A_{\varphi \circ \psi}^{\Theta \circ \psi}$ by (6) (by precomposing with a rotation, we may also assume that $0<z_{1}<1$ if we wish). By Lemma 2.1, it follows that

$$
\begin{aligned}
\left\langle\mathbf{x}_{n}, \mathbf{x}_{i}\right\rangle\left\langle\mathbf{x}_{i}, \mathbf{x}_{j}\right\rangle\left\langle\mathbf{x}_{j}, \mathbf{x}_{n}\right\rangle & =\left\langle\tilde{k}_{0}, \tilde{k}_{z_{i}}\right\rangle\left\langle\tilde{k}_{z_{i}}, \tilde{k}_{z_{j}}\right\rangle\left\langle\tilde{k}_{z_{j}}, \tilde{k}_{0}\right\rangle \\
& =\left\langle k_{z_{i}}, k_{0}\right\rangle\left\langle k_{z_{j}}, k_{z_{i}}\right\rangle\left\langle k_{0}, k_{z_{i}}\right\rangle \\
& =\frac{\left(1-\left|z_{i}\right|^{2}\right)\left(1-\left|z_{j}\right|^{2}\right)}{1-\overline{z_{j}} z_{i}}
\end{aligned}
$$

for $1 \leq i, j \leq n$, which is the desired condition (3).

$(\Leftarrow)$ Suppose that there exist distinct points $z_{1}, z_{2}, \ldots, z_{n-1}$ in $\mathbb{D}$ such that (3) holds and let $\Theta$ be a Blasckhe product of order $n$ having its zeros at the points 
$z_{1}, z_{2}, \ldots, z_{n-1}$ and $z_{n}=0$. It follows from (3) that

$$
\left\langle\mathbf{x}_{n}, \mathbf{x}_{i}\right\rangle\left\langle\mathbf{x}_{i}, \mathbf{x}_{j}\right\rangle\left\langle\mathbf{x}_{j}, \mathbf{x}_{n}\right\rangle=\left\langle\tilde{k}_{0}, \tilde{k}_{z_{i}}\right\rangle\left\langle\tilde{k}_{z_{i}}, \tilde{k}_{z_{j}}\right\rangle\left\langle\tilde{k}_{z_{j}}, \tilde{k}_{0}\right\rangle
$$

for $1 \leq i, j \leq n$, whence by Lemma 2.1 there exists a unitary operator $U: \mathbb{C}^{n} \rightarrow \mathcal{K}_{\Theta}$ and unimodular constants $\alpha_{1}, \alpha_{2}, \ldots, \alpha_{n}$ such that $U x_{i}=\alpha_{i} \tilde{k}_{z_{i}}$ for $1 \leq i \leq n$. If $\varphi$ is a polynomial satisfying $\varphi\left(z_{i}\right)=\lambda_{i}$ for $1 \leq i \leq n$, then $A_{\varphi}^{\Theta} \tilde{k}_{z_{i}}=\lambda_{i} \tilde{k}_{z_{i}}$, whence

$$
U M \mathbf{x}_{i}=\lambda_{i} U \mathbf{x}_{i}=\varphi\left(z_{i}\right) \alpha_{i} \tilde{k}_{z_{i}}=\alpha_{i} A_{\varphi}^{\Theta} \tilde{k}_{z_{i}}=A_{\varphi}^{\Theta} U \mathbf{x}_{i}
$$

for $1 \leq i \leq n$ so that $M \cong A_{\varphi}^{\Theta}$.

\section{Hyperbolic geometry}

It is easy to construct matrices which are not unitarily equivalent to any analytic truncated Toeplitz operator. For instance, any matrix having a pair of orthogonal eigenvectors suffices. On the other hand, what can be said about matrices having distinct eigenvalues and such that no pair of eigenvectors is orthogonal? Using some basic hyperbolic geometry, we can construct a family of such matrices which are not unitarily equivalent to an analytic truncated Toeplitz operator.

Example 1. Let us begin by searching for a matrix $M \in \mathbf{M}_{n}(\mathbb{C})$ having distinct eigenvalues and whose corresponding normalized eigenvectors $\mathbf{x}_{1}, \mathbf{x}_{2}, \ldots, \mathbf{x}_{n}$ are such that condition (3) does not hold for any points $z_{1}, z_{2}, \ldots, z_{n}$ in $\mathbb{D}$. Fix $0<g<1$ and let $G$ be the $n \times n$ matrix with entries

$$
G_{i j}= \begin{cases}1 & \text { if } i=j \\ g & \text { if } i \neq j\end{cases}
$$

Since $G=g \mathbf{u u}^{*}+(1-g) I$, where $\mathbf{u}=(1,1, \ldots, 1)$ and $I$ denotes the $n \times n$ identity matrix, it follows that the eigenvalues of $G$ are precisely

$$
\underbrace{1-g, \quad 1-g, \ldots, \quad 1-g}_{n-1 \text { times }}, \quad 1+(n-1) g,
$$

whence $G$ is positive definite. A routine computation confirms that the entries of the positive square root $X$ of $G$ are given by

$$
X_{i j}= \begin{cases}\frac{(n-1) \sqrt{1-g}+\sqrt{1+(n-1) g}}{n} & \text { if } i=j, \\ \frac{-\sqrt{1-g}+\sqrt{1+(n-1) g}}{n} & \text { if } i \neq j,\end{cases}
$$

and hence each column $\mathbf{x}_{i}$ of $X=\left(\mathbf{x}_{1}\left|\mathbf{x}_{2}\right| \cdots \mid \mathbf{x}_{n}\right)$ is a unit vector. If $\lambda_{1}, \lambda_{2}, \ldots, \lambda_{n}$ are distinct complex numbers and $D=\operatorname{diag}\left(\lambda_{1}, \lambda_{2}, \ldots, \lambda_{n}\right)$, then the matrix $M=$ $X D X^{-1}$ satisfies $X^{*} X=G$ and $M \mathbf{x}_{i}=\lambda_{i} \mathbf{x}_{i}$ for $1 \leq i \leq n$.

Suppose toward a contradiction that $M$ is unitarily equivalent to $A_{\varphi}^{\Theta}$ for some $\varphi \in H^{\infty}$ and some Blaschke product $\Theta$ having distinct zeros $z_{1}, z_{2}, \ldots, z_{n}$ (the hypothesis that $D$ has distinct eigenvalues ensures that the $z_{i}$ are distinct). By (5) it follows that $\rho\left(z_{i}, z_{j}\right)=\sqrt{1-g^{2}}$ for $i \neq j$. Since the hyperbolic metric (also called the Poincaré metric) on $\mathbb{D}$ satisfies

$$
\psi(z, w):=\log \frac{1+\rho(z, w)}{1-\rho(z, w)},
$$


it follows that

$$
\psi\left(z_{i}, z_{j}\right)= \begin{cases}r & \text { if } i \neq j \\ 0 & \text { if } i=j\end{cases}
$$

where $r=2 \tanh ^{-1} \sqrt{1-g^{2}}$ [19, pp. 4-5]. This is impossible if $n \geq 5$. Indeed, suppose that $z_{1}, z_{2}, z_{3}, z_{4}, z_{5}$ satisfy (8). Recalling that a pseudohyperbolic circle is also a Euclidean circle [19, p. 3], we obtain circles $\Gamma_{1}, \Gamma_{2}$, both of the same pseudohyperbolic radius, such that $z_{1}, z_{3}, z_{4}, z_{5} \in \Gamma_{2}$ and $z_{2}, z_{3}, z_{4}, z_{5} \in \Gamma_{1}$. This implies that $\left\{z_{3}, z_{4}, z_{5}\right\} \subseteq \Gamma_{1} \cap \Gamma_{2}$, which contradicts the fact that two distinct Euclidean circles can meet in at most two points.

A slightly more complicated proof shows that (8) is also impossible if $n=4$. Although the corresponding result is obvious in the Euclidean plane, to prove it in the hyperbolic plane one first recalls that the Poincaré model of the hyperbolic plane satisfies Hilbert's axioms [20, Sect. 39]. One can then prove that Propositions I.2I.22 and I.24-I.28 of Euclid's Elements [10] can be obtained in the Poincaré model [20, Thm. 10.4] and then proceed as in the Euclidean case.

It actually turns out that any matrix $3 \times 3$ or larger produced using the method of Example 1 cannot be a complex symmetric operator. In particular, no such matrix can be unitarily equivalent to a truncated Toeplitz operator, analytic or otherwise. First note that the entries of $W=X^{-1}$ are given by

$$
W_{i j}= \begin{cases}\frac{1}{n}\left(\frac{n-1}{\sqrt{1-g}}+\frac{1}{\sqrt{1+(n-1) g}}\right) & \text { if } i=j, \\ \frac{1}{n}\left(-\frac{1}{\sqrt{1-g}}+\frac{1}{\sqrt{1+(n-1) g}}\right) & \text { if } i \neq j .\end{cases}
$$

Writing $W=\left(\mathbf{w}_{1}\left|\mathbf{w}_{2}\right| \cdots \mid \mathbf{w}_{n}\right)$ in column-by-column format, we find that

$$
\left\|\mathbf{w}_{i}\right\|=\sqrt{\frac{1+(n-2) g}{1+[(n-2)-(n-1) g] g}}
$$

for $i=1,2, \ldots, n$. Upon dividing $W$ by the preceding quantity we obtain the matrix $Y=\left(\mathbf{y}_{1}\left|\mathbf{y}_{2}\right| \cdots \mid \mathbf{y}_{n}\right)$ whose entries are given by

$$
Y_{i j}= \begin{cases}\frac{\sqrt{1-g}+(n-1) \sqrt{1+(n-1) g}}{n \sqrt{1+(n-2) g}} & \text { if } i=j, \\ \frac{\sqrt{1-g}-\sqrt{1+(n-1) g}}{n \sqrt{1+(n-2) g}} & \text { if } i \neq j,\end{cases}
$$

and whose columns $\mathbf{y}_{1}, \mathbf{y}_{2}, \ldots, \mathbf{y}_{n}$ are unit vectors. Since $M=X D X^{-1}$ and $X$ is self-adjoint, it follows that $M^{*}=Y \bar{D} Y^{-1}$ Therefore $\mathbf{y}_{1}, \mathbf{y}_{2}, \ldots, \mathbf{y}_{n}$ are unit eigenvectors of $M^{*}$ corresponding to the eigenvalues $\overline{\lambda_{1}}, \overline{\lambda_{2}}, \ldots, \overline{\lambda_{n}}$. If $n \geq 3$, then

$$
\left|\left\langle\mathbf{x}_{i}, \mathbf{x}_{j}\right\rangle\right|=g \neq \frac{g}{1+(n-2) g}=\left|\left\langle\mathbf{y}_{i}, \mathbf{y}_{j}\right\rangle\right|,
$$

whence $M$ is not a complex symmetric operator by [3, Thm. 1].

\section{THE $2 \times 2$ CASE}

If $M \in \mathbf{M}_{2}(\mathbb{C})$ and $\Theta$ is a Blaschke product of order two, then there exists a truncated Toeplitz operator on $\mathcal{K}_{\Theta}$ which is unitarily equivalent to $M$ [8, Thm. 5.2]. However, if one insists upon using analytic symbols, then things are quite different. 
Corollary 4.1. If $M \in \mathbf{M}_{2}(\mathbb{C})$, then $M$ is unitarily equivalent to an analytic truncated Toeplitz operator if and only if either

(i) $M$ is a multiple of the identity or

(ii) $M$ is not normal.

Proof. $(\Rightarrow)$ Suppose that $M \cong A_{\varphi}^{\Theta}$ for some Blaschke product $\Theta$ of order two and some $\varphi$ in $H^{\infty}$.

Case 1. If $\Theta$ has distinct zeros $z_{1}, z_{2}$, then $\tilde{k}_{z_{1}}$ and $\tilde{k}_{z_{2}}$ are linearly independent eigenvectors of $A_{\varphi}$ corresponding to the eigenvalues $\varphi\left(z_{1}\right)$ and $\varphi\left(z_{2}\right)$, respectively. In particular, $M$ is diagonalizable. If $\varphi\left(z_{1}\right)=\varphi\left(z_{2}\right)$, then $M$ is a multiple of the identity. If $\varphi\left(z_{1}\right) \neq \varphi\left(z_{2}\right)$, then $A_{\varphi}$ is not normal since $\left\langle\tilde{k}_{z_{1}}, \tilde{k}_{z_{2}}\right\rangle \neq 0$.

Case 2. If $\Theta$ has a repeated root, then by (6) we may assume that $\Theta(z)=z^{2}$. Thus $M$ is unitarily equivalent to a lower-triangular Toeplitz matrix. Since a normal triangular matrix is diagonal [2, p. 133], it follows that $M$ is either nonnormal or a multiple of the identity matrix.

$(\Leftarrow)$ If $M$ is a multiple of the identity matrix, then there is nothing to prove. Suppose now that $M$ is not normal. If $M$ has repeated eigenvalues, then Schur's theorem on unitary triangularization [21, Thm. 2.3.1] asserts that $M$ is unitarily equivalent to a lower triangular Toeplitz matrix and hence an analytic truncated Toeplitz operator. Suppose that $M$ has distinct eigenvalues with corresponding unit eigenvectors $\mathbf{x}_{1}$ and $\mathbf{x}_{2}$. The only nontrivial condition in (3) that needs to be satisfied is $1-\left|z_{1}\right|^{2}=\left|\left\langle\mathbf{x}_{2}, \mathbf{x}_{1}\right\rangle\right|^{2} \neq 0$, which has many solutions.

Example 2. Consider the nonnormal matrix

$$
M=\left(\begin{array}{ll}
1 & 2 \\
0 & 3
\end{array}\right),
$$

whose eigenvalues are $\lambda_{1}=1$ and $\lambda_{2}=3$. Corresponding unit eigenvectors of $M$ corresponding to these eigenvalues are

$$
\mathbf{x}_{1}=(1,0), \quad \mathbf{x}_{2}=\left(\frac{1}{\sqrt{2}}, \frac{1}{\sqrt{2}}\right) .
$$

Guided by the proof of Theorem 1.1, we set

$$
z_{1}=\sqrt{1-\left|\left\langle\mathbf{x}_{2}, \mathbf{x}_{1}\right\rangle\right|^{2}}=\frac{1}{\sqrt{2}}
$$

and $z_{2}=0$. Next, we search for a polynomial $\varphi(z)$ such that

$$
\varphi\left(\frac{1}{\sqrt{2}}\right)=1, \quad \varphi(0)=3 .
$$

One such polynomial is $\varphi(z)=3-2 \sqrt{2} z$, from which we conclude that $M$ is unitarily equivalent to $A_{\varphi}^{\Theta}$, where

$$
\Theta(z)=z\left(\frac{z-\frac{1}{\sqrt{2}}}{1-\frac{1}{\sqrt{2}} z}\right) .
$$

Following J. Tener [32, we say that a matrix is UECSM if it is unitarily equivalent to a complex symmetric matrix (i.e., it represents a complex symmetric operator with respect to some orthonormal basis). Although there are many proofs of the following result (see [3, Cor. 3], 6, Cor. 3.3], [13, Ex. 6], 12, [16, [17, Cor. 1], 22. p. 477], or [32, Cor. 3]), we feel compelled to provide yet another.

Corollary 4.2. If $M \in \mathbf{M}_{2}(\mathbb{C})$, then $M$ is UECSM. 
Proof. Let $M$ be a $2 \times 2$ matrix. If $M$ is either a multiple of the identity or normal, then $M$ is trivially UECSM (by the Spectral Theorem). Otherwise, $M$ is unitarily equivalent to an analytic truncated Toeplitz operator by (4.1) and hence UECSM [11, 13.

\section{THE $3 \times 3$ CASE}

Although the $3 \times 3$ case is significantly more complicated than the $2 \times 2$ case, we are still able to arrive at a complete solution, including a simple computational criterion (9). Moreover, we also show that every $3 \times 3$ complex symmetric matrix is unitarily equivalent to a direct sum of truncated Toeplitz operators (Theorem 5.2).

Theorem 5.1. If $M \in \mathbf{M}_{3}(\mathbb{C})$ has distinct eigenvalues $\lambda_{1}, \lambda_{2}, \lambda_{3}$ with corresponding normalized eigenvectors $\mathbf{x}_{1}, \mathbf{x}_{2}, \mathbf{x}_{3}$ satisfying $\left\langle\mathbf{x}_{i}, \mathbf{x}_{j}\right\rangle \neq 0$ for $1 \leq i, j \leq 3$, then the following are equivalent:

(i) $M$ is unitarily equivalent to an analytic truncated Toeplitz operator.

(ii) $M$ is unitarily equivalent to a complex symmetric matrix.

(iii) The condition

$$
\operatorname{det} X^{*} X=\left(1-\left|\left\langle\mathbf{x}_{1}, \mathbf{x}_{2}\right\rangle\right|^{2}\right)\left(1-\left|\left\langle\mathbf{x}_{2}, \mathbf{x}_{3}\right\rangle\right|^{2}\right)\left(1-\left|\left\langle\mathbf{x}_{3}, \mathbf{x}_{1}\right\rangle\right|^{2}\right)
$$

holds, where $X=\left(\mathbf{x}_{1}\left|\mathbf{x}_{2}\right| \mathbf{x}_{3}\right)$ is the matrix having $\mathbf{x}_{1}, \mathbf{x}_{2}, \mathbf{x}_{3}$ as its columns.

Proof. (i) $\Rightarrow$ (ii) This implication is well-known [11, 13, 26] (i.e., every truncated Toeplitz operator is a complex symmetric operator).

(ii) $\Rightarrow$ (iii). Without loss of generality, we may assume that $M=M^{t}$ is a complex symmetric matrix. In this case $\overline{\mathbf{x}_{1}}, \overline{\mathbf{x}_{2}}, \overline{\mathbf{x}_{3}}$ are unit eigenvectors of $M^{*}$ corresponding to the eigenvalues $\overline{\lambda_{1}}, \overline{\lambda_{2}}, \overline{\lambda_{3}}$. In light of the fact that

$$
\lambda_{i}\left\langle\mathbf{x}_{i}, \overline{\mathbf{x}_{j}}\right\rangle=\left\langle M \mathbf{x}_{i}, \overline{\mathbf{x}_{j}}\right\rangle=\left\langle\mathbf{x}_{i}, M^{*} \overline{\mathbf{x}_{j}}\right\rangle=\left\langle\mathbf{x}_{i}, \overline{M \mathbf{x}_{j}}\right\rangle=\left\langle\mathbf{x}_{i}, \overline{\lambda_{j} \mathbf{x}_{j}}\right\rangle=\lambda_{j}\left\langle\mathbf{x}_{i}, \overline{\mathbf{x}_{j}}\right\rangle,
$$

we see that $\left\langle\mathbf{x}_{i}, \overline{\mathbf{x}_{j}}\right\rangle=0$ for $i \neq j$.

We claim that

$$
\begin{aligned}
& \left|\left\langle\mathbf{x}_{1}, \overline{\mathbf{x}_{1}}\right\rangle\right|^{2}=\left(1-\left|\left\langle\mathbf{x}_{1}, \mathbf{x}_{2}\right\rangle\right|^{2}\right)\left(1-\left|\left\langle\mathbf{x}_{3}, \mathbf{x}_{1}\right\rangle\right|^{2}\right), \\
& \left|\left\langle\mathbf{x}_{2}, \overline{\mathbf{x}_{2}}\right\rangle\right|^{2}=\left(1-\left|\left\langle\mathbf{x}_{1}, \mathbf{x}_{2}\right\rangle\right|^{2}\right)\left(1-\left|\left\langle\mathbf{x}_{2}, \mathbf{x}_{3}\right\rangle\right|^{2}\right), \\
& \left|\left\langle\mathbf{x}_{3}, \overline{\mathbf{x}_{3}}\right\rangle\right|^{2}=\left(1-\left|\left\langle\mathbf{x}_{2}, \mathbf{x}_{3}\right\rangle\right|^{2}\right)\left(1-\left|\left\langle\mathbf{x}_{3}, \mathbf{x}_{1}\right\rangle\right|^{2}\right) .
\end{aligned}
$$

We prove only (11), since (10) and (12) can be proven using a similar method. First note that $\overline{\mathbf{x}_{1}}$ and $\overline{\mathbf{x}_{2}}$ are linearly independent since $\lambda_{1} \neq \lambda_{2}$. Since $\left\langle\mathbf{x}_{3}, \overline{\mathbf{x}_{1}}\right\rangle=$ $\left\langle\mathbf{x}_{3}, \overline{\mathbf{x}_{2}}\right\rangle=0$, it follows that $\left\{\overline{\mathbf{x}}_{1}, \overline{\mathbf{x}}_{2}, \mathbf{x}_{3}\right\}$ is a basis for $\mathbb{C}^{3}$. Thus the basis $\left\{\mathbf{e}_{1}, \mathbf{e}_{2}, \mathbf{e}_{3}\right\}$ defined by setting $\mathbf{e}_{1}=\overline{\mathbf{x}_{1}}, \mathbf{e}_{3}=\mathbf{x}_{3}$, and

$$
\mathbf{e}_{2}=\frac{\overline{\mathbf{x}_{2}}-\left\langle\overline{\mathbf{x}_{2}}, \mathbf{e}_{1}\right\rangle \mathbf{e}_{1}}{\left\|\overline{\mathbf{x}_{2}}-\left\langle\overline{\mathbf{x}_{2}}, \mathbf{e}_{1}\right\rangle \mathbf{e}_{1}\right\|}=\frac{\overline{\mathbf{x}_{2}}-\left\langle\mathbf{x}_{1}, \mathbf{x}_{2}\right\rangle \overline{\mathbf{x}_{1}}}{\sqrt{1-\left|\left\langle\mathbf{x}_{1}, \mathbf{x}_{2}\right\rangle\right|^{2}}}
$$

is orthonormal. Since $\mathbf{x}_{2}$ is a unit vector it follows that

$$
\begin{aligned}
1 & =\left|\left\langle\mathbf{x}_{2}, \mathbf{e}_{1}\right\rangle\right|^{2}+\left|\left\langle\mathbf{x}_{2}, \mathbf{e}_{2}\right\rangle\right|^{2}+\left|\left\langle\mathbf{x}_{2}, \mathbf{e}_{3}\right\rangle\right|^{2} \\
& =\left|\left\langle\mathbf{x}_{2}, \overline{\mathbf{x}_{1}}\right\rangle\right|^{2}+\left|\left\langle\mathbf{x}_{2}, \frac{\overline{\mathbf{x}_{2}}-\left\langle\mathbf{x}_{1}, \mathbf{x}_{2}\right\rangle \overline{\mathbf{x}_{1}}}{\sqrt{1-\left|\left\langle\mathbf{x}_{1}, \mathbf{x}_{2}\right\rangle\right|^{2}}}\right\rangle\right|^{2}+\left|\left\langle\mathbf{x}_{2}, \mathbf{x}_{3}\right\rangle\right|^{2} \\
& =\frac{\left|\left\langle\mathbf{x}_{2}, \overline{\mathbf{x}_{2}}\right\rangle\right|^{2}}{1-\left|\left\langle\mathbf{x}_{1}, \mathbf{x}_{2}\right\rangle\right|^{2}}+\left|\left\langle\mathbf{x}_{2}, \mathbf{x}_{3}\right\rangle\right|^{2},
\end{aligned}
$$

which is equivalent to (11). 
Since $X^{t} X=\operatorname{diag}\left(\left\langle\mathbf{x}_{1}, \overline{\mathbf{x}_{1}}\right\rangle,\left\langle\mathbf{x}_{2}, \overline{\mathbf{x}_{2}}\right\rangle,\left\langle\mathbf{x}_{3}, \overline{\mathbf{x}_{3}}\right\rangle\right)$, it follows from (10), (11), and (12) that

$$
\begin{aligned}
\operatorname{det} X^{*} X & =|\operatorname{det} X|^{2} \\
& =\left|\operatorname{det} X^{t} X\right| \\
& =\left|\left\langle\mathbf{x}_{1}, \overline{\mathbf{x}_{1}}\right\rangle\left\langle\mathbf{x}_{2}, \overline{\mathbf{x}_{2}}\right\rangle\left\langle\mathbf{x}_{3}, \overline{\mathbf{x}_{3}}\right\rangle\right| \\
& =\left(1-\left|\left\langle\mathbf{x}_{1}, \mathbf{x}_{2}\right\rangle\right|^{2}\right)\left(1-\left|\left\langle\mathbf{x}_{2}, \mathbf{x}_{3}\right\rangle\right|^{2}\right)\left(1-\left|\left\langle\mathbf{x}_{3}, \mathbf{x}_{1}\right\rangle\right|^{2}\right) .
\end{aligned}
$$

This yields the desired condition (9).

(iii) $\Leftrightarrow$ (i). In light of Theorem 1.1 and its proof, it follows that $M$ is unitarily equivalent to an analytic truncated Toeplitz operator if and only if there exist $z_{1} \in(0,1)$ and $z_{2} \in \mathbb{D}$ such that (3) holds for $1 \leq i, j, k \leq 3$. Moreover, by (4) we know that $z_{1}$ and $z_{2}$ must be given by

$$
z_{1}=\sqrt{1-\left|\left\langle\mathbf{x}_{1}, \mathbf{x}_{3}\right\rangle\right|^{2}}, \quad z_{2}=\sqrt{1-\left|\left\langle\mathbf{x}_{2}, \mathbf{x}_{3}\right\rangle\right|^{2}} e^{i t}
$$

for some $t \in \mathbb{R}$. In other words, $M$ is unitarily equivalent to an analytic truncated Toeplitz operator if and only if there exists a real number $t$ such that the numbers $z_{1}, z_{2}$, as defined by (13), satisfy (3):

$$
\left\langle\mathbf{x}_{3}, \mathbf{x}_{1}\right\rangle\left\langle\mathbf{x}_{1}, \mathbf{x}_{2}\right\rangle\left\langle\mathbf{x}_{2}, \mathbf{x}_{3}\right\rangle=\frac{\left|\left\langle\mathbf{x}_{1}, \mathbf{x}_{3}\right\rangle\right|^{2}\left|\left\langle\mathbf{x}_{2}, \mathbf{x}_{3}\right\rangle\right|^{2}}{1-\overline{z_{2}} z_{1}}
$$

which is equivalent to

$$
\overline{z_{2}} z_{1}=1-\frac{\left\langle\mathbf{x}_{1}, \mathbf{x}_{3}\right\rangle\left\langle\mathbf{x}_{3}, \mathbf{x}_{2}\right\rangle}{\left\langle\mathbf{x}_{1}, \mathbf{x}_{2}\right\rangle}
$$

Substituting (13) into the preceding equation we obtain

$$
e^{-i t} \sqrt{1-\left|\left\langle\mathbf{x}_{1}, \mathbf{x}_{3}\right\rangle\right|^{2}} \sqrt{1-\left|\left\langle\mathbf{x}_{2}, \mathbf{x}_{3}\right\rangle\right|^{2}}=1-\frac{\left\langle\mathbf{x}_{1}, \mathbf{x}_{3}\right\rangle\left\langle\mathbf{x}_{3}, \mathbf{x}_{2}\right\rangle}{\left\langle\mathbf{x}_{1}, \mathbf{x}_{2}\right\rangle},
$$

which has a solution $t \in \mathbb{R}$ if and only if

$$
\left(1-\left|\left\langle\mathbf{x}_{1}, \mathbf{x}_{3}\right\rangle\right|^{2}\right)\left(1-\left|\left\langle\mathbf{x}_{2}, \mathbf{x}_{3}\right\rangle\right|^{2}\right)=\left|1-\frac{\left\langle\mathbf{x}_{1}, \mathbf{x}_{3}\right\rangle\left\langle\mathbf{x}_{3}, \mathbf{x}_{2}\right\rangle}{\left\langle\mathbf{x}_{1}, \mathbf{x}_{2}\right\rangle}\right|^{2} .
$$

Expanding the preceding, we obtain

$$
\begin{aligned}
& \left|\left\langle\mathbf{x}_{1}, \mathbf{x}_{3}\right\rangle\left\langle\mathbf{x}_{3}, \mathbf{x}_{2}\right\rangle\right|^{2}+\left|\left\langle\mathbf{x}_{2}, \mathbf{x}_{1}\right\rangle\left\langle\mathbf{x}_{1}, \mathbf{x}_{3}\right\rangle\right|^{2}+\left|\left\langle\mathbf{x}_{3}, \mathbf{x}_{2}\right\rangle\left\langle\mathbf{x}_{2}, \mathbf{x}_{1}\right\rangle\right|^{2} \\
& \quad=\left|\left\langle\mathbf{x}_{1}, \mathbf{x}_{2}\right\rangle\left\langle\mathbf{x}_{2}, \mathbf{x}_{3}\right\rangle\left\langle\mathbf{x}_{3}, \mathbf{x}_{1}\right\rangle\right|^{2}+2 \operatorname{Re}\left\langle\mathbf{x}_{1}, \mathbf{x}_{2}\right\rangle\left\langle\mathbf{x}_{2}, \mathbf{x}_{3}\right\rangle\left\langle\mathbf{x}_{3}, \mathbf{x}_{1}\right\rangle .
\end{aligned}
$$

Adding the quantity

$$
1-\left|\left\langle\mathbf{x}_{1}, \mathbf{x}_{2}\right\rangle\right|^{2}-\left|\left\langle\mathbf{x}_{2}, \mathbf{x}_{3}\right\rangle\right|^{2}-\left|\left\langle\mathbf{x}_{3}, \mathbf{x}_{1}\right\rangle\right|^{2}
$$

to both sides of (14) yields

$$
\begin{array}{r}
1-\left|\left\langle\mathbf{x}_{1}, \mathbf{x}_{2}\right\rangle\right|^{2}-\left|\left\langle\mathbf{x}_{2}, \mathbf{x}_{3}\right\rangle\right|^{2}-\left|\left\langle\mathbf{x}_{3}, \mathbf{x}_{1}\right\rangle\right|^{2}+2 \operatorname{Re}\left\langle\mathbf{x}_{1}, \mathbf{x}_{2}\right\rangle\left\langle\mathbf{x}_{2}, \mathbf{x}_{3}\right\rangle\left\langle\mathbf{x}_{3}, \mathbf{x}_{1}\right\rangle \\
=\left(1-\left|\left\langle\mathbf{x}_{1}, \mathbf{x}_{2}\right\rangle\right|^{2}\right)\left(1-\left|\left\langle\mathbf{x}_{2}, \mathbf{x}_{3}\right\rangle\right|^{2}\right)\left(1-\left|\left\langle\mathbf{x}_{3}, \mathbf{x}_{1}\right\rangle\right|^{2}\right),
\end{array}
$$

which is equivalent to (9).

Theorem 5.2. If $M \in \mathbf{M}_{3}(\mathbb{C})$ is complex symmetric, then $M$ is unitarily equivalent to a direct sum of truncated Toeplitz operators. 
In order to proceed with the proof of Theorem [5.2 we require the following lemma from [16. The proof in the preprint [16] is long and involved, since it requires invoking the fact that a matrix $7 \times 7$ or smaller which is unitarily equivalent to its transpose is UECSM (this can fail for matrices $8 \times 8$ and larger). We provide a much simpler proof below.

Lemma 5.3. If $M \in \mathbf{M}_{3}(\mathbb{C})$, then $M$ is UECSM if and only if

$$
\operatorname{tr} M^{*} M^{2} M^{* 2} M=\operatorname{tr} M M^{* 2} M^{2} M^{*} \text {. }
$$

Proof. By Corollary 4.2 we may assume that $M$ is irreducible since otherwise $M$ is obviously UECSM. We use the term irreducible in the operator-theoretic sense. Namely, a matrix $T \in M_{n}(\mathbb{C})$ is called irreducible if $T$ is not unitarily equivalent to a direct sum $A \oplus B$, where $A \in M_{d}(\mathbb{C})$ and $B \in M_{n-d}(\mathbb{C})$ for some $1<d<n$. Equivalently, $T$ is irreducible if and only if the only normal matrices commuting with $T$ are multiples of the identity.

We claim that $M$ is UECSM if and only if $M \cong M^{t}$. One direction is simple, for if $M$ is UECSM, then there exists a unitary matrix $U$ such that $U^{*} M U=\left(U^{*} M U\right)^{t}$. In other words, $M\left(U U^{t}\right)=\left(U U^{t}\right) M^{t}$, whence $M \cong M^{t}$.

On the other hand, if $M \cong M^{t}$, then we may write $M=U M^{t} U^{*}$, where $U$ is unitary. It follows that $M^{t}=\bar{U} M U^{t}$, whence $M U \bar{U}=U \bar{U} M$. Since $M$ is irreducible, $U \bar{U}=\alpha I$ for some unimodular constant $\alpha$. The preceding implies that $U=\alpha U^{t}$, whence $\alpha^{2}=1$. However, $\alpha=-1$ is impossible since every skewsymmetric matrix of odd dimension is singular. Therefore $U=U^{t}$ is symmetric and unitary. By Takagi's Factorization Theorem [21, we may write $U=V V^{t}$, where $V$ is unitary and whence $V^{*} M V=\left(V^{*} M V\right)^{t}$. In other words, $M$ is UECSM.

Having shown that $M$ is UECSM if and only if $M \cong M^{t}$, we need only show that (15) holds if and only if $M \cong M^{t}$. To this end, we recall that a refinement by Sibirskiur [30] of a well-known result of Pearcy [24] asserts that $A, B \in \mathbf{M}_{3}(\mathbb{C})$ are unitarily equivalent if and only if $\Phi(A)=\Phi(B)$, where $\Phi: M_{3}(\mathbb{C}) \rightarrow \mathbb{C}^{7}$ is the function defined by

$$
\Phi(X)=\left(\operatorname{tr} X, \operatorname{tr} X^{2}, \operatorname{tr} X^{3}, \operatorname{tr} X^{*} X, \operatorname{tr} X^{*} X^{2}, \operatorname{tr} X^{* 2} X^{2}, \operatorname{tr} X^{*} X^{2} X^{* 2} X\right) .
$$

It is easy to see that the first six traces in (16) are automatically equal for $X=M$ and $X=M^{t}$ [16. In other words, $M \cong M^{t}$ if and only if $\operatorname{tr} X^{*} X^{2} X^{* 2} X$ yields the same value for $X=M$ and $X=M^{t}$. Using standard properties of the trace and transpose, one sees that this condition is equivalent to (15).

With the preceding lemma in hand, we are now ready to prove Theorem 5.2 .

Proof of Theorem 5.2. Suppose that $M$ is a $3 \times 3$ matrix. After possibly scaling and adding a multiple of the identity, up to unitary equivalence $M$ falls into precisely one of the following classes:

$$
\underbrace{\left(\begin{array}{lll}
0 & 0 & 0 \\
a & 0 & 0 \\
b & c & 0
\end{array}\right)}_{\text {one distinct eigenvalue }}, \quad \underbrace{\left(\begin{array}{ccc}
0 & 0 & 0 \\
a & 0 & 0 \\
b & c & 1
\end{array}\right)}_{\text {two distinct eigenvalues }}, \underbrace{\left(\begin{array}{lll}
0 & 0 & 0 \\
a & 1 & 0 \\
b & c & \lambda
\end{array}\right)}_{\text {three distinct eigenvalues }},
$$

where $a, b, c \in \mathbb{C}$ and $\lambda \neq 0,1$. 
Case 1. If $M$ has one distinct eigenvalue, then without loss of generality we may assume that $M$ is of the form of the first matrix listed in (17). Using Lemma 5.3 and Mathematica it follows that $M$ is UECSM if and only if

$$
|a|^{2}|c|^{2}\left(|a|^{2}-|c|^{2}\right)=0
$$

(see [17, Ex. 1] and [32, Ex. 1] for other approaches). In other words, $M$ is UECSM if and only if either (i) $a=0$, (ii) $c=0$, or (iii) $|a|=|c|$. If either $a=0$ or $c=0$, then $M$ has rank one, whence $M$ is unitarily equivalent to a truncated Toeplitz operator by [ $[$, Thm. 5.1]. On the other hand, if $|a|=|c|$, then conjugating $M$ by a diagonal unitary matrix shows that $M$ is unitarily equivalent to a Toeplitz matrix and hence $M$ represents a truncated Toeplitz operator on $\mathcal{K}_{z^{3}}$ with respect to the orthonormal basis $\left\{1, z, z^{2}\right\}$.

Case 2. If $M$ has exactly two distinct eigenvalues, then we may assume that $M$ is of the form of the second matrix listed in (17). Using Lemma 5.3 and Mathematica, it follows that $M$ is UECSM if and only if either (i) $a=0$, (ii) $b=c=0$, or (iii) $a, c \neq 0$ and

$$
|b+a c|^{2}=|c|^{2}\left(1+|c|^{2}\right)
$$

If $a=0$, then $M$ has rank one, whence $M$ is unitarily equivalent to a truncated Toeplitz operator by [8, Thm. 5.1]. If $b=c=0$, then $M$ is the direct sum of a $2 \times 2$ and a $1 \times 1$ matrix. By [8, Thm. 5.2], it follows that $M$ is unitarily equivalent to the direct sum of truncated Toeplitz operators.

The third case is more difficult to handle. Suppose that $a, c \neq 0$ and that (18) holds. Upon conjugating $M$ by a suitable diagonal unitary matrix, we may further assume that $c>0$ and $b+a c \geq 0$. Let $\Theta$ denote the Blaschke product

$$
\Theta(z)=z^{2}\left(\frac{z-r}{1-r z}\right)
$$

which has a double root at 0 and a simple zero at $r \in(0,1)$, which is to be determined. An orthonormal basis $\left\{e_{1}, e_{2}, e_{3}\right\}$ for $\mathcal{K}_{\Theta}$ is given by

$$
e_{1}(z)=1, \quad e_{2}(z)=z, \quad e_{3}(z)=z^{2} \frac{\sqrt{1-r^{2}}}{1-r z} .
$$

If $\varphi(z)=\alpha z+\beta z^{2}$, then the matrix for $A_{\varphi}^{\Theta}$ with respect to the basis (19) is given by

$$
\left(\begin{array}{ccc}
0 & 0 & 0 \\
\alpha & 0 & 0 \\
\beta \sqrt{1-r^{2}} & (\alpha+\beta r) \sqrt{1-r^{2}} & r(\alpha+\beta r)
\end{array}\right) .
$$

At this point it is easily verified that for

$$
\alpha=a, \quad \beta=\frac{b}{c} \sqrt{1+c^{2}}, \quad r=\frac{1}{\sqrt{1+c^{2}}},
$$

the matrix (20) is precisely $M$. In other words, $M$ is unitarily equivalent to an analytic truncated Toeplitz operator on $\mathcal{K}_{\Theta}$.

Case 3. Suppose that $M$ has three distinct eigenvalues. If $M$ has a pair of eigenvectors which are orthogonal, then we may assume that $M$ is of the form of the third matrix listed in (17) where $c=0$. In this case, Lemma 5.3 and Mathematica tell us that $M$ is UECSM if and only if $|a b|^{2}|\lambda-1|^{2}=0$. Since $\lambda \neq 1$ it follows 
that either $a=0$ or $b=0$. Both cases lead to the conclusion that $M$ is unitarily equivalent to the direct sum of a $2 \times 2$ and a $1 \times 1$ matrix. In particular, $M$ is unitarily equivalent to a direct sum of truncated Toeplitz operators.

If $M$ has no pair of nonzero eigenvectors which are orthogonal to each other, then we may appeal to Theorem 5.1 to conclude that $M$ is unitarily equivalent to an analytic truncated Toeplitz operator.

\section{A NECESSARY CONDITION}

Recall that every truncated Toeplitz operator is a complex symmetric operator and hence UECSM [11, 13, 26. Thus we might as well start with a complex symmetric matrix $M$ in the first place. Unlike Theorem 1.1, the following proposition is phrased completely in terms of the initial data $\mathbf{x}_{1}, \mathbf{x}_{2}, \ldots, \mathbf{x}_{n}$. In particular, there is no mention whatsoever of the unknowns $z_{1}, z_{2}, \ldots, z_{n-1}$.

Corollary 6.1. Suppose that $M \in \mathbf{M}_{n}(\mathbb{C})$ is complex symmetric and has distinct eigenvalues with corresponding unit eigenvectors $\mathbf{x}_{1}, \mathbf{x}_{2}, \ldots, \mathbf{x}_{n}$ satisfying $\left\langle\mathbf{x}_{i}, \mathbf{x}_{j}\right\rangle \neq$ 0 for $i \neq j$. The condition

$$
\left|\left\langle\mathbf{x}_{i}, \overline{\mathbf{x}_{i}}\right\rangle\right|^{2}=\prod_{\substack{j=1 \\ j \neq i}}^{n}\left(1-\left|\left\langle\mathbf{x}_{j}, \mathbf{x}_{i}\right\rangle\right|^{2}\right)
$$

for $i=1,2, \ldots, n$ is necessary for $M$ to be unitarily equivalent to an analytic truncated Toeplitz operator. If $n \leq 3$, then the preceding condition is also sufficient.

Proof. Maintaining the notation and conventions of Theorem 1.1 and its proof, let

$$
\Theta(z)=\prod_{i=1}^{n} \frac{z-z_{i}}{1-\overline{z_{i}} z}
$$

and observe that

$$
\prod_{\substack{j=1 \\ j \neq i}}^{n}\left(1-\left|\left\langle\mathbf{x}_{i}, \mathbf{x}_{j}\right\rangle\right|^{2}\right)=\prod_{\substack{j=1 \\ j \neq i}}^{n} \rho^{2}\left(z_{i}, z_{j}\right)=\left(1-\left|z_{i}\right|^{2}\right)^{2}\left|\Theta^{\prime}\left(z_{i}\right)\right|^{2}
$$

holds by (5). Next note that the hypothesis upon the eigenvectors of $M$ implies that $M$ is irreducible. Since $M=M^{t}$, it follows that $M=J M^{*} J$, where $J$ denotes the canonical conjugation

$$
J\left(\zeta_{1}, \zeta_{2}, \ldots, \zeta_{n}\right)=\left(\overline{\zeta_{1}}, \overline{\zeta_{2}}, \ldots, \overline{\zeta_{n}}\right)
$$

on $\mathbb{C}^{n}$. If $J^{\prime}$ is another conjugation which satisfies $M=J^{\prime} M^{*} J^{\prime}$, then $J J^{\prime}$ is unitary and commutes with $M$, whence $J J^{\prime}$ is a multiple of the identity. Thus $J$ is the unique conjugation, up to a unimodular constant factor, which satisfies $M=J M^{t} J$.

Now suppose that $U M=A_{\varphi}^{\Theta} U$ for some unitary $U: \mathbb{C}^{n} \rightarrow \mathcal{K}_{\Theta}$. Since $A_{\varphi}^{\Theta}$ is irreducible, a similar argument shows that the conjugation $C: \mathcal{K}_{\Theta} \rightarrow \mathcal{K}_{\Theta}$ defined by $C f=\overline{f z} \Theta$ is the unique conjugation, up to a unimodular constant factor, such that $A_{\varphi}^{\Theta}=C\left(A_{\varphi}^{\Theta}\right)^{*} C\left[11,13\right.$, 26]. Since $A_{\varphi}^{\Theta}=\left(U J U^{*}\right)\left(A_{\varphi}^{\Theta}\right)^{*}\left(U J U^{*}\right)$ and $U J U^{*}$ is a conjugation on $\mathcal{K}_{\Theta}$, it follows that $U J U^{*}=\gamma C$ for some unimodular constant $\gamma$. 
This yields

$$
\begin{aligned}
\left|\left\langle\mathbf{x}_{i}, \overline{\mathbf{x}_{i}}\right\rangle\right|^{2} & =\left|\left\langle\mathbf{x}_{i}, J \mathbf{x}_{i}\right\rangle\right|^{2}=\left|\left\langle U \mathbf{x}_{i}, U J \mathbf{x}_{i}\right\rangle\right|^{2} \\
& =\left|\left\langle U \mathbf{x}_{i}, C U \mathbf{x}_{i}\right\rangle\right|^{2}=\left|\left\langle\tilde{k}_{z_{i}}, k_{z_{i}}\right\rangle\right|^{2} \\
& =\left(1-\left|z_{i}\right|^{2}\right)^{2}\left|\Theta^{\prime}\left(z_{i}\right)\right|^{2} \\
& =\prod_{j=1}^{n}\left(1-\left|\left\langle\mathbf{x}_{j}, \mathbf{x}_{i}\right\rangle\right|^{2}\right)
\end{aligned}
$$

by (22).

To see that (21) is sufficient in the $3 \times 3$ case, simply observe that (21) implies (10), (11), and (12). In other words, (21) implies that (14) holds, whence $M$ is unitarily equivalent to an analytic truncated Toeplitz operator by Theorem 5.1 .

\section{OPEN PROBLEMS}

Although there has been a recent surge in activity devoted to truncated Toeplitz operators under unitary equivalence $7,8,31$, there are still many basic questions left unanswered. We conclude this paper with a series of open problems suggested by this work.

Question 1. Is every complex symmetric matrix $M \in \mathbf{M}_{n}(\mathbb{C})$ unitarily equivalent to a direct sum of truncated Toeplitz operators?

In other words, are truncated Toeplitz operators the basic building blocks of complex symmetric operators? For $n \geq 4$ numerical evidence strongly suggests that the implication (i) $\Leftrightarrow$ (ii) of Theorem 5.1 fails generically. In particular, (21) tends to fail for all $i=1,2, \ldots, n$ for randomly generated complex symmetric matrices $M \in \mathbf{M}_{n}(\mathbb{C})$ which satisfy the hypotheses of Corollary 6.1. On the other hand, nothing that we know of prevents such a matrix from being unitarily equivalent to a truncated Toeplitz operator with a symbol in $L^{\infty}$ (as opposed to $H^{\infty}$ ).

Question 2. Let $n \geq 4$. Is every complex symmetric matrix $M \in \mathbf{M}_{n}(\mathbb{C})$ having no pair of orthogonal, nonzero eigenvectors unitarily equivalent to a truncated Toeplitz operator?

A variant of the preceding is:

Question 3. Let $n \geq 4$. Is every irreducible complex symmetric matrix $M \in$ $\mathbf{M}_{n}(\mathbb{C})$ unitarily equivalent to a truncated Toeplitz operator?

Recently, the first author and J. Tener [16] showed that every complex symmetric matrix is unitarily equivalent to a direct sum of (i) irreducible complex symmetric matrices or (ii) matrices of the form $A \oplus A^{t}$, where $A$ is irreducible and not UECSM (such matrices are necessarily $6 \times 6$ or larger). This immediately suggests the following question.

Question 4. For $A \in \mathbf{M}_{n}(\mathbb{C})$, is the matrix $A \oplus A^{t} \in \mathbf{M}_{2 n}(\mathbb{C})$ unitarily equivalent to a direct sum of truncated Toeplitz operators?

Let $S$ denote the unilateral shift and recall that $S$ is not a complex symmetric operator [11, Ex. 2.14], [13, Prop. 1], [17, Thm. 4], 18, Cor. 7]. On the other hand, $T=S^{*} \oplus S$ is a complex symmetric operator [14, Ex. 5] which appears to be a 
promising candidate for a counterexample to Question 4 in the infinite-dimensional setting.

One method for producing complex symmetric matrix representations of a given truncated Toeplitz operator is to use modified Aleksandrov-Clark bases for $\mathcal{K}_{\Theta}$. We refer the reader to [15, Sect. 2.3, 5.2] for specific details.

Question 5. Suppose that $M \in \mathbf{M}_{n}(\mathbb{C})$ is complex symmetric. If $M$ is unitarily equivalent to a truncated Toeplitz operator, does there exist an inner function $\Theta$, a symbol $\varphi \in L^{\infty}$, and a modified Aleksandrov-Clark basis $\beta$ for $\mathcal{K}_{\Theta}$ such that $M$ is the matrix representation of $A_{\varphi}^{\Theta}$ with respect to the basis $\beta$ ?

In other words, do all such unitary equivalences between complex symmetric matrices and truncated Toeplitz operators arise essentially from Aleksandrov-Clark representations?

\section{ACKNOWLEDGMENTS}

The authors relied heavily upon numerical experiments to test several conjectures (which eventually led to proofs of Theorems 5.1 and 5.2). The authors wish to thank J. Tener for independently confirming several of their numerical observations.

\section{REFERENCES}

1. Jim Agler and John E. McCarthy, Pick interpolation and Hilbert function spaces, Graduate Studies in Mathematics, vol. 44, American Mathematical Society, Providence, RI, 2002. MR 1882259 (2003b:47001)

2. S. Axler, Linear algebra done right, second ed., Undergraduate Texts in Mathematics, Springer-Verlag, New York, 1997. MR1482226 (98i:15001)

3. L. Balayan and S. R. Garcia, Unitary equivalence to a complex symmetric matrix: Geometric criteria, Oper. Matrices 4 (2010), no. 1, 53-76. MR2655004

4. A. Baranov, Chalendar I., Fricain E., Mashreghi J. E., and D. Timotin, Bounded symbols and reproducing kernel thesis for truncated Toeplitz operators, J. Funct. Anal. 259 (2010), no. 10, 2673-2701. MR.2679022

5. I. Chalendar, E. Fricain, and D. Timotin, On an extremal problem of Garcia and Ross, Oper. Matrices 3 (2009), no. 4, 541-546. MR2597679 (2011b:30130)

6. Nicolas Chevrot, Emmanuel Fricain, and Dan Timotin, The characteristic function of a complex symmetric contraction, Proc. Amer. Math. Soc. 135 (2007), no. 9, 2877-2886 (electronic). MR2317964 (2008c:47025)

7. J. A. Cima, W. T. Ross, and W. R. Wogen, Truncated Toeplitz operators on finite dimensional spaces, Oper. Matrices 2 (2008), no. 3, 357-369. MR2440673 (2009e:47047)

8. Joseph A. Cima, Stephan Ramon Garcia, William T. Ross, and Warren R. Wogen, Truncated Toeplitz operators: Spatial isomorphism, unitary equivalence, and similarity, Indiana Univ. Math. J. 59 (2010), no. 2, 595-620. MR2648079

9. P. L. Duren, Theory of $H^{p}$ spaces, Academic Press, New York, 1970. MR0268655 (42:3552)

10. Euclid, Euclid's elements, Green Lion Press, Santa Fe, NM, 2002, all thirteen books complete in one volume, The Thomas L. Heath translation, edited by Dana Densmore. MR 1932864 (2003j:01044)

11. S. R. Garcia, Conjugation and Clark operators, Recent advances in operator-related function theory, Contemp. Math., vol. 393, Amer. Math. Soc., Providence, RI, 2006, pp. 67-111. MR 2198373 (2007b:47073)

12. S. R. Garcia, D. E. Poore, and M. K. Wyse, Unitary equivalence to a complex symmetric matrix: A modulus criterion, Oper. Matrices 5 (2011), no. 2, 273-287.

13. S. R. Garcia and M. Putinar, Complex symmetric operators and applications, Trans. Amer. Math. Soc. 358 (2006), no. 3, 1285-1315 (electronic). MR.2187654(2006j:47036)

14. _ Complex symmetric operators and applications. II, Trans. Amer. Math. Soc. 359 (2007), no. 8, 3913-3931 (electronic). MR2302518 (2008b:47005) 
15. S. R. Garcia and W. T. Ross, A nonlinear extremal problem on the Hardy space, Comp. Methods. Function Theory 9 (2009), no. 2, 485-524. MR2572653(2010k:47062)

16. S. R. Garcia and J. E. Tener, Unitary equivalence of a matrix to its transpose, J. Operator Theory (to appear). http://arxiv.org/abs/0908.2107.

17. S. R. Garcia and W. R. Wogen, Some new classes of complex symmetric operators, Trans. Amer. Math. Soc. 362 (2010), no. 11, 6065-6077. MR.2661508

18. Stephan Ramon Garcia, Means of unitaries, conjugations, and the Friedrichs operator, J. Math. Anal. Appl. 335 (2007), no. 2, 941-947. MR2345511 (2008i:47070)

19. J. Garnett, Bounded analytic functions, first ed., Graduate Texts in Mathematics, vol. 236, Springer, New York, 2007. MR2261424 (2007e:30049)

20. R. Hartshorne, Geometry: Euclid and beyond, Undergraduate Texts in Mathematics, Springer-Verlag, New York, 2000. MR.1761093(2001h:51001)

21. Roger A. Horn and Charles R. Johnson, Matrix analysis, Cambridge University Press, Cambridge, 1990. Corrected reprint of the 1985 original. MR1084815 (91i:15001)

22. Alan McIntosh, The Toeplitz-Hausdorff theorem and ellipticity conditions, Amer. Math. Monthly 85 (1978), no. 6, 475-477. MR0506368 (58:22127)

23. N. Nikolski, Operators, functions, and systems: An easy reading. Vol. 2, Mathematical Surveys and Monographs, vol. 93, American Mathematical Society, Providence, RI, 2002. Model operators and systems, translated from the French by Andreas Hartmann and revised by the author. MR1892647(2003i:47001b)

24. Carl Pearcy, A complete set of unitary invariants for $3 \times 3$ complex matrices, Trans. Amer. Math. Soc. 104 (1962), 425-429. MR0144911 (26:2451)

25. D. Sarason, A remark on the Volterra operator, J. Math. Anal. Appl. 12 (1965), 244-246. MR $0192355(33: 580)$

26. Algebraic properties of truncated Toeplitz operators, Oper. Matrices 1 (2007), no. 4, 491-526. MR2363975 (2008i:47060)

27. _ Unbounded operators commuting with restricted backward shifts, Oper. Matrices 2 (2008), no. 4, 583-601. MR2468883 (2009k:47083)

28. U Unbounded Toeplitz operators, Integral Equations Operator Theory 61 (2008), no. 2, 281-298. MR2418122 (2010c:47073)

29. N. Sedlock, Algebras of truncated Toeplitz operators, Oper. Matrices 5 (2011), no. 2, 309-326.

30. K. S. Sibirskiū, A minimal polynomial basis of unitary invariants of a square matrix of order three, Mat. Zametki 3 (1968), 291-295. MR0228522 (37:4102)

31. E. Strouse, D. Timotin, and M. Zarrabi, Unitary equivalence to truncated Toeplitz operators (submitted). http://arxiv.org/abs/1011.6055

32. J. E. Tener, Unitary equivalence to a complex symmetric matrix: an algorithm, J. Math. Anal. Appl. 341 (2008), no. 1, 640-648. MR2394112 (2008m:15062)

33. Lloyd N. Trefethen and Mark Embree, Spectra and pseudospectra, Princeton University Press, Princeton, NJ, 2005. The behavior of nonnormal matrices and operators. MR2155029 (2006d:15001)

34. J. Vermeer, Orthogonal similarity of a real matrix and its transpose, Linear Algebra Appl. 428 (2008), no. 1, 382-392. MR.2372597 (2008m:15043)

Department of Mathematics, Pomona College, Claremont, California 91711

E-mail address: Stephan.Garcia@pomona.edu

$U R L:$ http://pages.pomona.edu/ sg064747

Department of Mathematics, Pomona College, Claremont, California 91711

E-mail address: dep02007@mymail.pomona.edu

Department of Mathematics and Computer Science, University of Richmond, RichMOND, VIRGINIA 23173

E-mail address: wross@richmond.edu

$U R L:$ http://facultystaff.richmond.edu/ ${ }^{\text {wross }}$ 